\title{
Puerarin Inhibits oxLDL-Induced Macrophage Activation and Foam Cell Formation in Human THP1 Macrophage
}

\author{
Heng Zhang, ${ }^{1}$ Zhenhua Zhai, ${ }^{2}$ Hongyu Zhou, ${ }^{3}$ Yao Li, ${ }^{1}$ Xiaojie Li, ${ }^{1}$ Yuhan Lin, \\ Weihong Li, ${ }^{1}$ Yueping Shi, ${ }^{4}$ and Ming-Sheng Zhou ${ }^{1}$ \\ ${ }^{1}$ Department of Physiology, Liaoning Medical University, Jinzhou, Liaoning 121001, China \\ ${ }^{2}$ Department of Oncology, Cancer Center, Turmounesis and Microenvironment Laboratory, The First Affiliated Hospital, \\ Liaoning Medical University, Jinzhou, Liaoning 121001, China \\ ${ }^{3}$ Vagelos Scholars Program of the Molecular Life Sciences, University of Pennsylvania, Philadelphia, PA 19104, USA \\ ${ }^{4}$ Department of Chinese Medicine, The First Affiliated Hospital, Liaoning Medical University, Jinzhou, \\ Liaoning 121001, China \\ Correspondence should be addressed to Ming-Sheng Zhou; zhoums1963@163.com
}

Received 28 July 2015; Revised 17 September 2015; Accepted 4 October 2015

Academic Editor: Ziad Mallat

Copyright (C) 2015 Heng Zhang et al. This is an open access article distributed under the Creative Commons Attribution License, which permits unrestricted use, distribution, and reproduction in any medium, provided the original work is properly cited.

Puerarin, an isoflavone derived from Kudzu roots, has been widely used for treatment of cardiovascular and cerebral vascular diseases in China and other Asian countries. However, the underlying mechanisms are largely unknown. The present study investigated whether puerarin inhibited atherogenic lipid oxLDL-mediated macrophage activation and foam cell formation in human THP1 macrophage. Treatment with oxLDL significantly increased the mRNA expression of proinflammatory cytokines tumor necrosis factor $\alpha$ (TNF $\alpha, 160 \%$ ) and interleukin (IL) $1 \beta$ (13 fold) accompanied by upregulation of toll-like receptor 4 (TLR4, $165 \%)$ and the ratio of phospho- $\mathrm{I} \kappa \mathrm{B} \alpha / \mathrm{I} \kappa \mathrm{B} \alpha$ in THP1 macrophage. Puerarin dose-dependently prevented an increase in oxLDLinduced proinflammatory gene expression with downregulation of TLR 4 and the ratio of phospho-I $\kappa \mathrm{B} \alpha / \mathrm{I} \kappa \mathrm{B} \alpha$. Furthermore, puerarin prevented oxLDL-mediated lipid deposition and foam cell formation associated with downregulation of scavenger receptor CD36. Flow cytometry analysis showed that puerarin reduced the number of early apoptotic cells of macrophages induced by oxLDL. Our results show that puerarin has anti-inflammatory and antiatherogenic effects in vitro; the underlying mechanisms may involve the inhibition of TLR4/NF $\kappa$ B pathway and downregulation of CD36 expression. The results from the present study provide scientific evidence and may expand our armamentarium to use puerarin for prevention and treatment of cardiovascular and atherosclerotic diseases.

\section{Introduction}

Atherosclerosis, the primary cause of heart disease and stroke, is responsible for approximately $50 \%$ of all deaths in Western societies and is the leading cause of deaths worldwide [1]. Over the past decades, therapeutic options to treat atherosclerosis have been significantly improved $[2,3]$. However, there is still an enormous unmet need: after 3 years, $20 \%$ of patients with acute coronary syndrome suffer from recurrent myocardial infarction despite optional medial therapy. Thus novel therapies to prevent atherogenesis or treat atherosclerosis are urgently needed [4]. Natural products, such as Chinese herbal medicine, could be an ideal source to develop safe and effective agents for treatment of cardiovascular and atherosclerotic diseases [5].

Atherosclerosis is considered as a chronic inflammatory disease of the arterial wall characterized with inflammation, oxidative stress, and immune system activation [4]. Monocytes/macrophages play a central role in atherosclerosis through the accumulation of cholesterol and production of inflammatory mediators and cytokines [6, 7]. Early atherogenesis is characterized by the adherence of blood circulating monocytes to vascular endothelium, then by their migration to the subendothelial space, and further 
by activation into macrophages [8]. Macrophages via their scavenger receptors take up oxidized LDL (oxLDL,) and other lipids, undergo activation, and produce cytokines, matrix metalloproteinases, and reactive oxygen species (ROS) while continuing to accumulate lipids and differentiate into foam cells to form the early lesions that mature into atherosclerotic plaques [9].

Puerarin (daidzein 8-C-glucoside, $\mathrm{C}_{21} \mathrm{H}_{20} \mathrm{C}_{9}$ ) is a major isoflavonoid compound isolated from the Chinese herb Kudzu roots, which is known as Gegen, one of the most popular Chinese traditional medicine [10]. Puerarin is available in common foods and has a long history for the treatment of cardiovascular and cerebrovascular diseases, including coronary artery disease, heart failure, hypertension, and myocardial infarction $[10,11]$, in China and other Asian countries. It has been proposed that puerarin may exert beneficial effects on cardiovascular system, including vasodilation, antioxidant, anti-inflammation, and antiplatelet aggregation [12-14]. Recent studies suggest that puerarin may have antiatherogenic effects $[15,16]$. However, the underlying mechanisms remain largely unknown. The activated macrophages release of inflammatory cytokines and uptake of oxLDL forming foam cells are critical for the development of atherosclerotic plaque $[17,18]$. It is well known that the oxidative modified LDLs such as oxLDL have atherogenic effects [9]. The present study investigated whether puerarin inhibited atherogenic lipid oxLDL-induced macrophage activation and foam cell formation, two key steps for development of atherosclerosis, in human THP1 macrophages, and elucidated the potential mechanisms of puerarin's antiatherosclerosis.

\section{Methods}

2.1. Materials. Human THP1 monocytes and supplemental medium were purchased from ATCC (ATCC TIB 202, Manassas, VA). Rabbit polyclonal anti-phospho-I $\kappa \mathrm{B} \alpha, \mathrm{I} \kappa \mathrm{B} \alpha$, and CD36 antibodies were obtained from Santa Cruz Biotechnology (Santa Cruz, CA). Puerarin (98.9\% purity) was purchased from Sigma Inco. (St Louis). oxLDL was purchased from Shanghai Luwen Biotech Inc. (Shanghai, China) and was used within 6 weeks after it was made. All other chemicals were of the best grade available from commercial sources.

2.2. Cell Culture. Human THP1 monocytes were cultured in RPMI 1640 medium supplemented with 10\% fetal bovine serum (FBS), $10 \mathrm{mmol} / \mathrm{L}$ HEPES, $2 \mu \mathrm{mol} / \mathrm{L}$ L-glutamine, $1 \mu \mathrm{mol} / \mathrm{L}$ sodium pyruvate, $100 \mathrm{U} / \mathrm{mL}$ penicillin, $100 \mu \mathrm{g} / \mathrm{mL}$ streptomycin, and $0.05 \mathrm{mmol} / \mathrm{L} 2$-mercaptoethanol. The cells were cultured at $37^{\circ} \mathrm{C}, 95 \%$ humidity, and $5 \% \mathrm{CO}_{2}$ and used between passages 4 and 16. The cells were seeded in six-well plates $\left(5 \times 10^{5}\right.$ cells/well) and differentiated into macrophages by preincubation with $100 \mathrm{ng} / \mathrm{mL}$ phorbol 12-myristate 13acetate (PMA, Sigma) for 36 hours. The cells were starved in serum-free RPMI 1640 medium for 24 hours before the experiments were performed. The cells were incubated with vehicle (equal amount of culture medium) and oxLDL $(50 \mu \mathrm{g} / \mathrm{mL}$ ) with or without puerarin (from $10 \mu \mathrm{g} / \mathrm{mL}$ to $100 \mu \mathrm{g} / \mathrm{mL}$ ) for 24 hours.
2.3. Real-Time PCR. The cells were harvested in $1 \mathrm{~mL}$ TRIzol reagent and total RNA ( $2 \mu \mathrm{g})$ was reverse-transcribed using a superscript II RT first strand synthesis kit (Gibco, BRL) according to the manufacturer's instructions. Real-time PCR for tumor necrosis factor $\alpha$ (TNF $\alpha$, assay ID: Hs00174128m1) and interleukin $1 \beta$ (IL1 $\beta$, assay ID: Hs01336189-m1) was performed in $20 \mu \mathrm{L}$ reaction mixture containing an appropriately diluted ( $80 \mathrm{ng}$ ) cDNA solution, $0.1 \mu \mathrm{mol} / \mathrm{L}$ of each primer, $0.2 \mu \mathrm{mol} / \mathrm{L}$ probe, and PCR master mix assay kit (ABI) as previously described [19]. Relative quantities of each transcript were normalized by a housekeeping gene (GAPDH) and expressed as fold increase versus control. All primers were ordered through Life Technologies (ABI) online system with assay ID.

2.4. Western Blot Analyses. The cells were harvested with lysis buffer containing a protein inhibitor cocktail. Protein was quantified by Bio-Rad assay, and $30 \mu \mathrm{g}$ of total protein was first subjected to SDS-PAGE and then transferred to nitrocellulose membranes. The membranes were incubated with primary rabbit polyclonal anti-CD36, antitoll-like receptor 4 (TLR4), anti-phospho- $\mathrm{K} \kappa \mathrm{B} \alpha$, or anti$\mathrm{I} \kappa \mathrm{B} \alpha$ (Santa Cruz Biotechnology) followed by incubation with a peroxidase-conjugated secondary antibody for 1 hour. Equivalence of protein loading and transfer was confirmed by reblotting the samples with anti- $\beta$-actin antibody (Santa Cruz Biotechnology). Immune reactive bands were detected by chemiluminescence and quantified by densitometry. Relative quantities of each protein were normalized by $\beta$-actin and expressed as fold increase versus control.

2.5. Foam Cell Formation. THP1 macrophages were preincubated with or without puerarin (10 to $100 \mu \mathrm{g} / \mathrm{mL})$ in chamber slides for 1 hour followed by incubation with oxLDL $(50 \mu \mathrm{g} / \mathrm{mL})$ for 72 hours. Oil Red O staining was used to visualize lipid deposits and hematoxylin staining was used to show the nuclei as previously described [19]. The images were acquired using microscope Zeiss. Oil red staining intensity was measured by Image-Pro Plus and used for the quantitative analysis of the lipid accumulation in the foam cells.

2.6. Apoptosis Assay by Flow Cytometry Analysis. The cells were incubated with vehicle, oxLDL $(100 \mu \mathrm{g} / \mathrm{mL})$, or oxLDL plus puerarin $(100 \mu \mathrm{g} / \mathrm{mL})$ for 24 hours. Annexin V-FITC and PI staining were performed to detect early stage apoptosis in human THP1 macrophage followed by manufactory instruction. Briefly, the cells $\left(1 \times 10^{5}\right)$ in $100 \mu \mathrm{L}$ of the binding buffer (10 mM HEPES pH 7.4, $140 \mathrm{mM} \mathrm{NaCl}$, and $2.5 \mathrm{mM} \mathrm{CaCl}_{2}$ ) were mixed with $5 \mu \mathrm{L}$ of annexin $\mathrm{V}$-FITC (BD Bioscience) and $10 \mu \mathrm{L}$ of PI (BD Bioscience). After 15-minute incubation at room temperature in the dark, the samples were immediately analyzed by flow cytometry. The relative number of cells that were annexin V-positive and PI-negative (early apoptotic cells) was determined.

2.7. Data Analysis. The results were expressed as mean \pm standard error of the mean (SEM). Statistical analyses 
were performed by ANOVA with Bonferroni's correction for multiple comparisons. Significance was assumed at $P<0.05$.

\section{Results}

3.1. Puerarin Prevented oxLDL Upregulation of CD36 Expression. CD36 is a member of the scavenger receptor class B family and a major scavenger receptor to uptake oxLDL (more than $70 \%$ ) in human macrophage [20]. oxLDL is a major ligand of CD36 and plays an essential role in the pathogenesis of atherosclerotic plaque [9]. It has been shown that oxLDL upregulates CD36 expression in macrophages [20]. To investigate whether puerarin inhibits oxLDL upregulation of CD36 expression, we exposed THP1 macrophages to oxLDL $(50 \mu \mathrm{g} / \mathrm{mL})$ with different doses of puerarin $(10 \mu \mathrm{g} / \mathrm{mL}$ to $100 \mu \mathrm{g} / \mathrm{mL}$ ) for 24 hours. As shown in Figure 1, oxLDL increased the protein expression of CD36 (170\%), which was significantly inhibited by puerarin in a dose-dependent manner $(P<0.05)$.

\subsection{Puerarin Inhibited Macrophage Activation in Response} to Atherogenic Lipid oxLDL. The chronic stimulation of the innate immune system in response to endogenous ligands such as oxLDL is believed to be crucial to atherogenesis [21, 22]. Monocytes and macrophages have long been considered to be important immune effector cells that participate in host defense and the regulation of inflammation [8]. There are two major populations of macrophages based on their homeostatic activities and functions, inflammatory (M1) and tissue resident (M2) macrophages [23]. M1 macrophages are generated during cell-mediated immune responses [21, 23]. Activated M1 macrophages produce proinflammatory cytokines, including TNF $\alpha$, IL1 $\beta$, interferon $\gamma$, CXCL9, and inducible nitric oxide [23]. TNF $\alpha$ and IL1 $\beta$ are important M1 macrophage markers [23]. As shown in Figure 2, the mRNA expression of proinflammatory cytokines TNF $\alpha$ and IL1 $\beta$ was significantly increased in the cells treated with oxLDL. Puerarin prevented increase in the mRNA expression of these proinflammatory genes induced by oxLDL.

\subsection{Puerarin Suppressed oxLDL Activation of TLR4/NFאB Sig-} naling. It has been proposed that oxLDL induces inflammatory responses via activation of TLRs pathway [24]. As shown in Figure 3, oxLDL significantly increased the protein expression of TLR4, and puerarin dose-dependently suppressed oxLDL-induced TLR4 expression with maximal inhibition of $60 \%$ at $100 \mu \mathrm{g} / \mathrm{mL}$. $\mathrm{NF} \kappa \mathrm{B}$ is a downstream molecule of TLR4 in the macrophage innate immune response [24, 25]. $\mathrm{NF} \kappa \mathrm{B}$ activation is initiated from the phosphorylation of $\mathrm{I} \kappa \mathrm{B}$ and inactivation of $\mathrm{I} \kappa \mathrm{B}$ by phosphorylation and proteolysis leads to $\mathrm{NF} \kappa \mathrm{B}$ translocation to the nucleus to trigger gene transcription. Increased phosphor (Ser32)-I $\kappa \mathrm{B}$ or the ratio of phospho- $\mathrm{I} \kappa \mathrm{B} / \mathrm{I} \kappa \mathrm{B}$ is an index of $\mathrm{NF} \kappa \mathrm{B}$ activation [25]. As shown in Figure 3, oxLDL treatment significantly increased the expression of phospho- $\mathrm{I} \kappa \mathrm{B} \alpha$ with a slight reduction in the expression of $\mathrm{I} \kappa \mathrm{B} \alpha$; therefore, the ratio of phospho$\mathrm{I} \kappa \mathrm{B} \alpha / \mathrm{I} \kappa \mathrm{B} \alpha$ was increased in oxLDL-treated cells $(P<$ 0.05). Puerarin dose-dependently reduced the expression

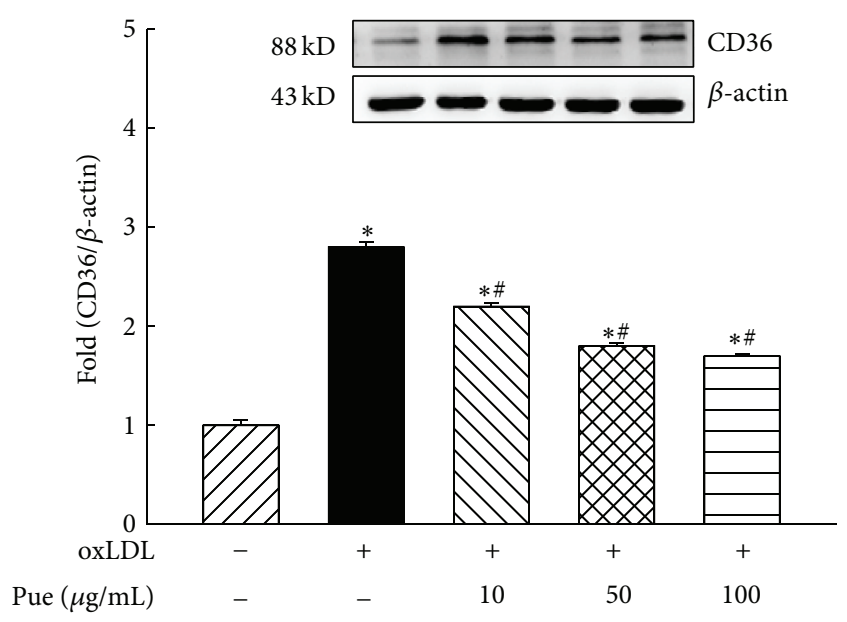

FIGURE 1: Effect of puerarin on the protein expression of CD36 in human THP1 macrophages. Incubation of oxLDL $(50 \mu \mathrm{g} / \mathrm{mL})$ for 24 hours significantly increased the protein expression of CD36, and puerarin dose-dependently reduced CD36 expression. Pue: puerarin. Data is expressed as mean \pm SEM. ${ }^{*} P<0.05$ versus control, ${ }^{\#} P<0.05$ versus oxLDL. $n=5$ to 6 .

of phospho- $\mathrm{I} \kappa \mathrm{B} \alpha$ and the ratio of phospho- $\mathrm{I} \kappa \mathrm{B} \alpha / \mathrm{I} \kappa \mathrm{B} \alpha$ in oxLDL-treated cells $(P<0.05)$.

3.4. Puerarin Inhibited oxLDL-Induced Foam Cell Formation. The macrophages uptake of oxLDL forming foam cell is a hallmark for atherosclerosis [18]. As shown in Figure 4, incubation of cells with oxLDL $(50 \mu \mathrm{g} / \mathrm{mL})$ for 72 hours resulted in lipid deposition in macrophages and foam cell formation. The quantitative analysis of oil red staining intensity showed that puerarin significantly reduced oil red staining intensity in a dose-dependent manner, confirming that puerarin inhibits oxLDL-induced foam cell formation.

3.5. Puerarin Inhibited oxLDL-Induced Early Apoptosis of Macrophage. Macrophages in atherosclerotic lesions have been shown to have cellular characteristics of both necrosis and apoptosis [26]. The majority of apoptotic cells in atherosclerotic lesions are macrophages localized near the necrotic areas of advanced lesions [26]. As shown in Figure 5, oxLDL significantly increased the cell number that was annexin V-positive and PI-negative (early apoptotic cells, $9.2 \pm 2.9 \%$ versus $2.47 \pm 0.8 \%$ in control, $P<0.05)$. Puerarin reduced the number of early apoptotic cells in the cell treated with oxLDL $(4.8 \pm 1.6 \%, P<0.05)$.

\section{Discussion}

Puerarin is a major isoflavonoid compound extracted from the Chinese medical herb Kudzu root [27, 28]. For more than 2000 years, Kudzu root has been used as a herbal medicine for the treatment of several diseases including fever, diabetes, and cardiovascular diseases in China and some Asian countries [10]. Currently, puerarin has been widely used for treatment 


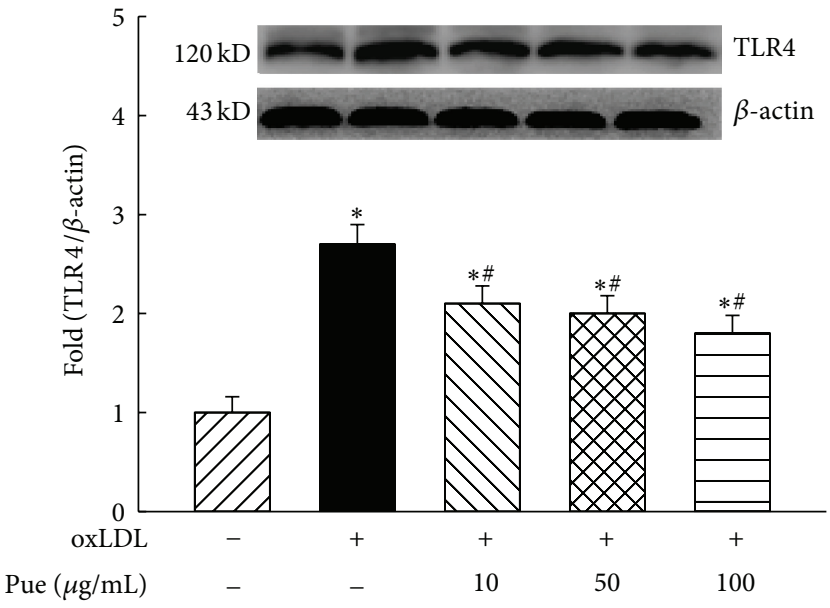

(a)

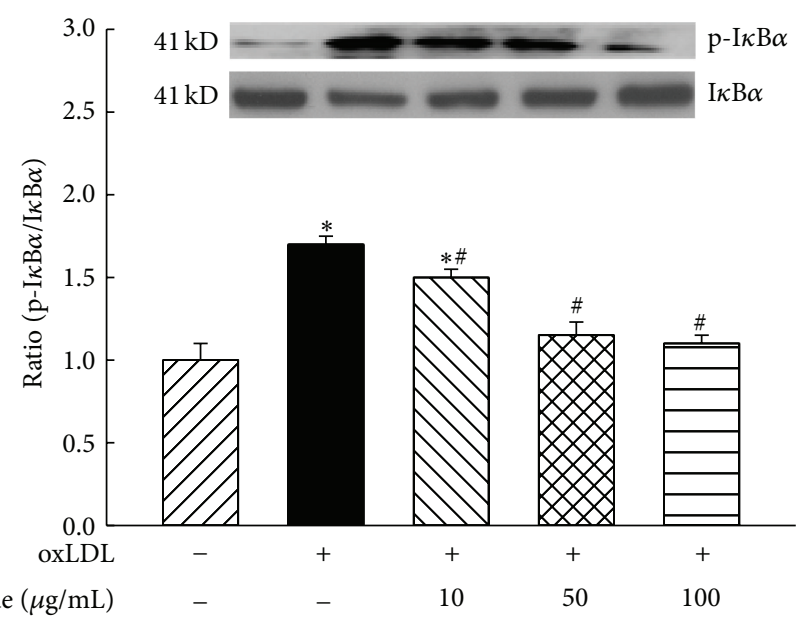

(b)

FIGURE 2: Effect of puerarin on the protein expression of toll-like receptor 4 (TLR4, (a)) and the ratio of phospho-(Ser32) I $\kappa \mathrm{B} \alpha / \mathrm{I} \kappa \mathrm{B} \alpha(\mathrm{b})$ in human THP1 macrophages. ${ }^{*} P<0.05$ versus control; ${ }^{*} P<0.05$ versus oxLDL. $n=5$ to 6 .

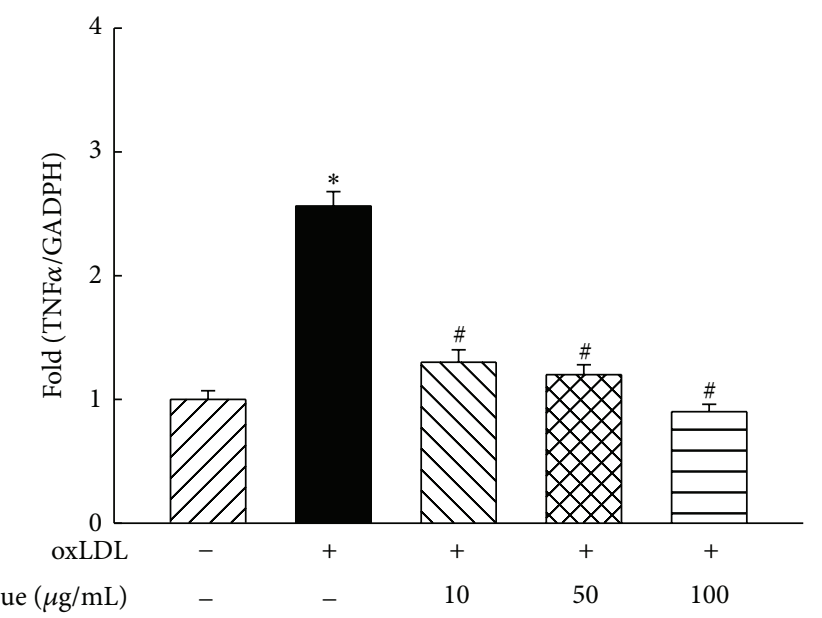

(a)

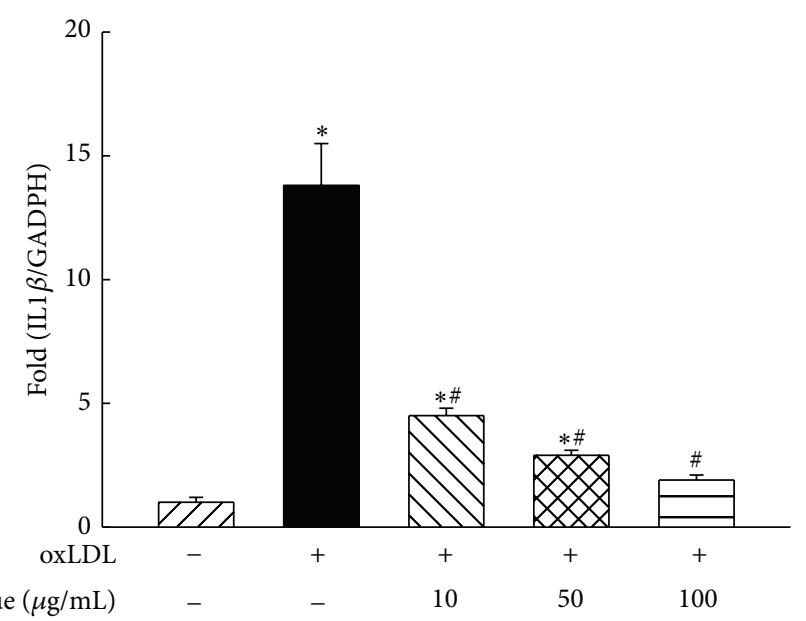

(b)

FIGURE 3: Effect of puerarin on the mRNA expression of tumor necrosis factor $\alpha$ (TNF $\alpha$, (a)) and interleukin $1 \beta$ (IL1 $\beta$, (b)) in human THP1 macrophages. ${ }^{*} P<0.05$ versus control; ${ }^{\#} P<0.05$ versus oxLDL. $n=5$ to 6 .

of coronary artery disease and angina pectoris in Chinese traditional medical practice $[10,28]$. A number of studies have shown that puerarin has cardiovascular protective effects such as antioxidant, vasorelaxation, and anti-inflammation and antiapoptosis in vascular endothelial cells $[13,14,28$, 29]. The present study demonstrated for the first time that in human THP1 macrophages puerarin dose-dependently suppressed atherogenic lipid oxLDL-induced macrophage activation and releases of inflammatory cytokines TNF $\alpha$ and IL1 $\beta$ associated with inhibition of innate immune pathway of TLR4/NFKB, and puerarin inhibited oxLDL-mediated lipid deposition and foam cell formation with downregulation of CD36 expression and inhibited oxLDL-induced macrophage apoptosis. These data strongly suggest that puerarin has important anti-inflammatory antiatherosclerotic effects through modulation of the innate immune cells.
It has been well established that the elevation of plasma level of oxLDLs is associated with an increased risk for the development of atherogenesis. During atherosclerosis, heightened oxidative stress in the artery wall gives rise to oxidized forms of LDL that provoke an inflammatory response [30]. The macrophages recognize and take up these oxidized lipids through a family of pattern recognition receptors known as scavenger receptors. CD36 is the predominant scavenger receptor for oxLDL uptake in macrophages [20]. Interaction of CD36 with oxLDL on macrophages triggers a signaling cascade response that is both proinflammatory and proatherogenic [24]. CD36 is believed to play a critical role in the initiation of atherosclerotic lesions through its ability to bind and internalize modified LDL trapped in the artery wall, facilitating the formation of macrophage foam cells and the release of inflammatory cytokines from 


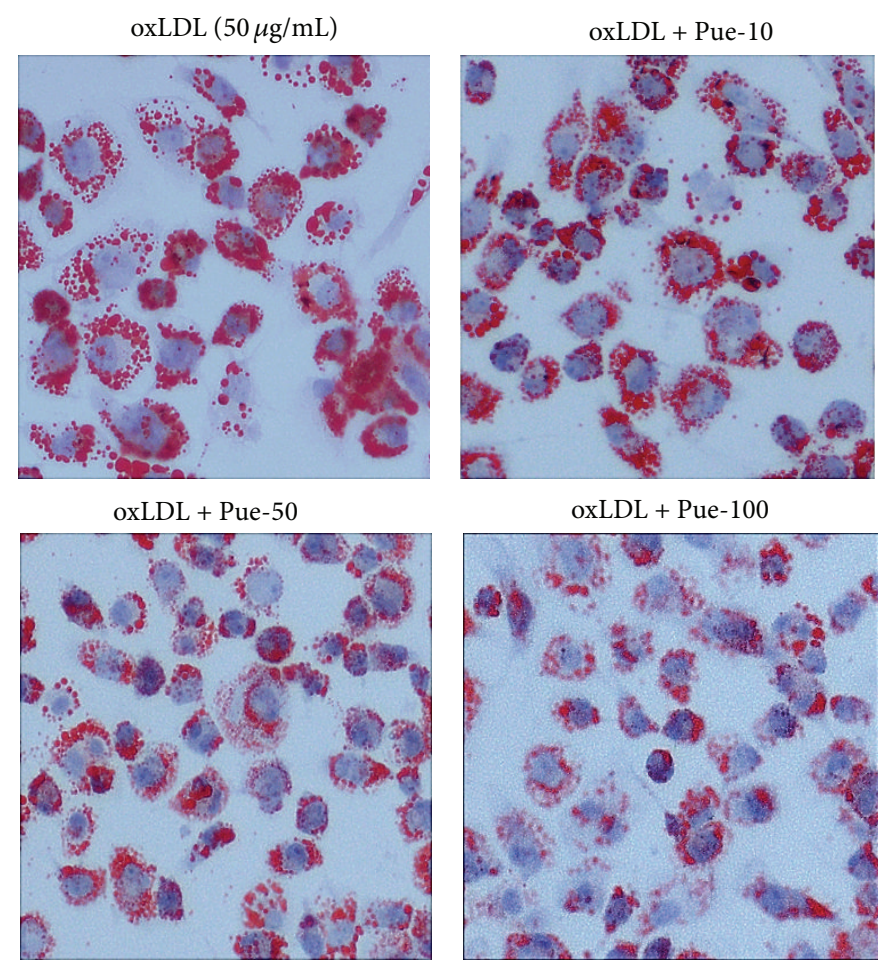

(a)

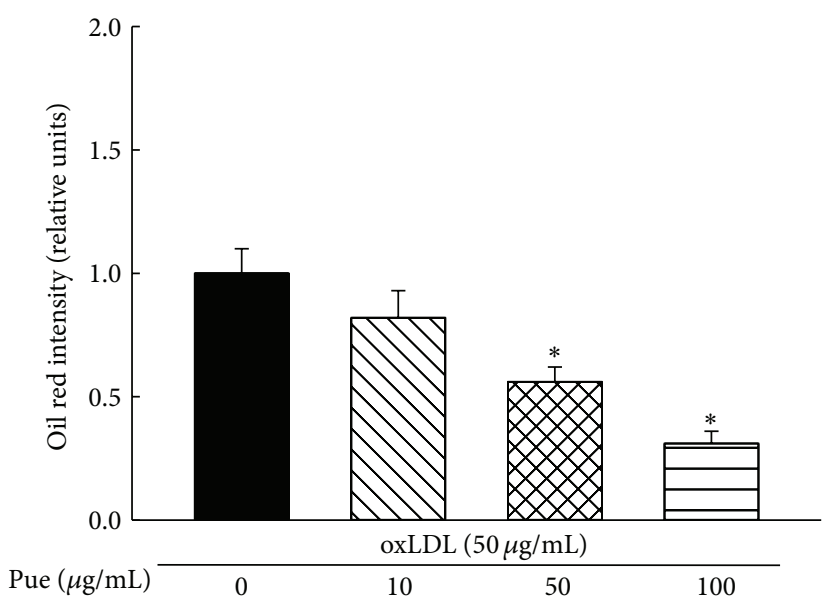

(b)

FIGURE 4: Effect of puerarin on oxLDL-induced foam cell formation in human THP1 macrophages. The THP1 macrophages were treated with oxLDL $(50 \mu \mathrm{g} / \mathrm{mL}$ ) with or without puerarin at various doses (from $10 \mu \mathrm{g} / \mathrm{mL}$ to $100 \mu \mathrm{g} / \mathrm{mL}$ ) for 72 hours. Oil red staining was performed, and oil red staining intensity was measured for the quantitation of the lipid accumulation in the foam cells. (a): representative images; (b): oil red intensity was quantified. ${ }^{*} P<0.05$ versus oxLDL. $n=5$.

lipid-loaded macrophages in the arteries [9]. The present study demonstrated that oxLDL significantly increased the expression of CD36 in human THP1 macrophages, and puerarin dose-dependently inhibited oxLDL upregulation of CD36 expression accompanied by a reduction in foam cell formation. Since CD36 is critical for oxLDL uptake and foam cell formation in macrophage $[9,24,31]$, other scavenger receptors such as scavenger receptor $\mathrm{A}$ and oxidized lowdensity lipoprotein receptor 1 (LOX1) may also participate in these processes mediated by oxLDL [24]. However, our previous study has shown that oxLDL did not affect the expression of scavenger receptor A and LOX1 in these cell lines and nicotine promoted foam cells and atherosclerosis via upregulation and stimulation of macrophage CD36 signaling [19]. Therefore, we speculate that inhibition of foam cell formation by puerarin may be at least in part attributed to its suppression of CD36 expression.

Inflammation plays a crucial role in the pathogenesis of atherosclerosis [4]. Inflammatory cytokines are uniformly more present in patients with atherosclerosis. Sites of atherosclerotic plaque development in the arterial wall are characterized by cholesterol accumulation and by peripheral blood monocytes and macrophage infiltration [22]. It is generally accepted that circulating inflammatory monocytes have a high capacity to migrate to tissues including the vascular wall, where they differentiate into inflammatory macrophages and foam cells in response to oxidized LDL [23]. The present study demonstrated that THP1 macrophages treated with oxLDL exhibited a significant increase in the mRNA expression of inflammatory cytokines TNF $\alpha$ and IL1 $\beta$, two cytokine markers for inflammatory M1 macrophage; puerarin prevented an increase in oxLDLinduced macrophage release of the cytokines, suggesting that puerarin inhibits macrophage activation with antiinflammatory effects.

It has been shown that oxLDL stimulates macrophage activation via the activation of TLRs/NF $\kappa$ B immune pathway [24]. A recent elegant study demonstrated that oxLDL, after binding to CD36, facilitated heterodimer formation of TLR4 and TLR6 in macrophages, and this coreceptor complex activated downstream molecule redoxsensitive transcription factor $\mathrm{NF} \kappa \mathrm{B}$, consequently resulting in macrophage activation and release/production of inflammatory chemokines and cytokines [24]. Puerarin has been reported to inhibit TLR4 innate signaling pathway in cerebral ischemia/reperfusion-induced tissue [32]. Here we showed that puerarin inhibited oxLDL-induced macrophages release of inflammatory cytokines associated with reduction in expression of TLR 4 and ratio of phosphor- $\mathrm{I} \kappa \mathrm{B} \alpha / \mathrm{I} \kappa \mathrm{B} \alpha$, suggesting that puerarin inhibits oxLDL-mediated macrophage 

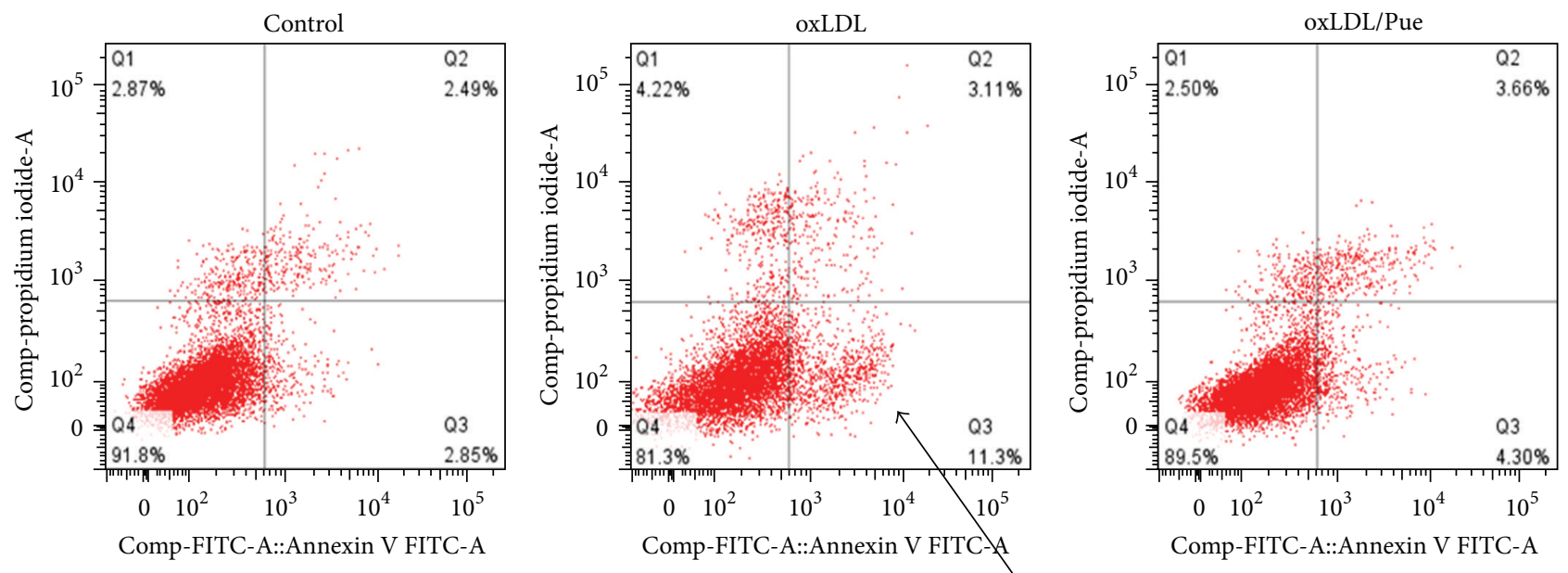

Early apoptotic cells

(a)

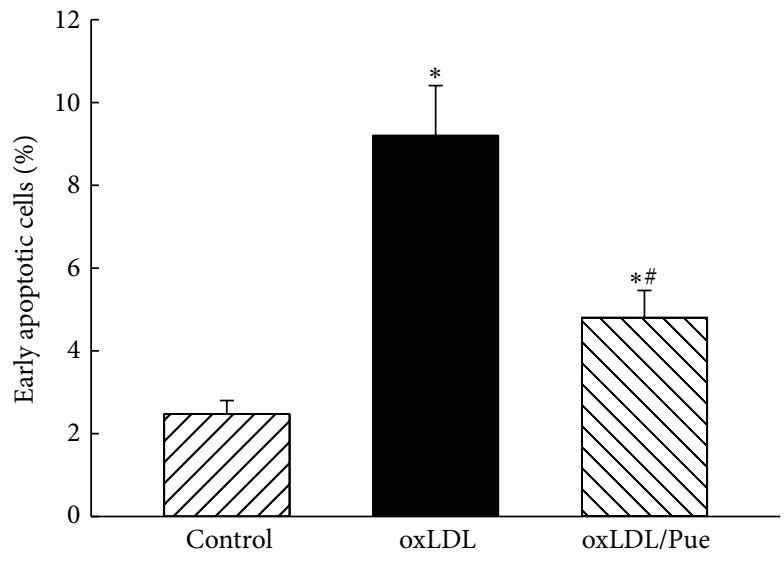

(b)

FIGURE 5: Effect of puerarin on oxLDL-induced early apoptotic cells in human THP1 macrophages. oxLDL ( $50 \mu \mathrm{g} / \mathrm{mL})$ significantly increased early apoptotic cells of macrophage (Annexin V-positive and PI-negative cells as indicated by arrow) determined by flow cytometry analysis, and treatment with puerarin $(100 \mu \mathrm{g} / \mathrm{mL})$ significantly reduced oxLDL-induced apoptotic cells. (a): representative original trace of flow cytometry; (b): the quantitative analysis of early apoptotic cells of macrophages in bar graphs. $P<0.05$ versus control; ${ }^{\#} P<0.05$ versus oxLDL. $n=6$.

activation via inhibition of TLR4/NFkB pathway. In the unstimulated cells, $\mathrm{NF} \kappa \mathrm{B}$ is present as an inactive, $\mathrm{I} \kappa \mathrm{B}$ bound complex in the cytoplasm. Many signals that lead to activation of $\mathrm{NF} \kappa \mathrm{B}$ converge on the ROS-dependent activation of $\mathrm{I} \kappa \mathrm{B}$ kinase (IKK) [33]. Activation of the IKK complex leads to the phosphorylation and degradation of $\mathrm{I} \kappa \mathrm{B}$, consequently activating $\mathrm{NF} \kappa \mathrm{B}[25,33]$. Puerarin is an isoflavone with potent antioxidant effect and may inhibit $\mathrm{NF} \kappa \mathrm{B}$ activation through suppression of ROS-dependent activation of IKK. In addition, our data showed that puerarin inhibited oxLDL-induced CD36 and TLR4 expression, and oxLDL can facilitate the interaction between CD36 and TLR4 to induce $\mathrm{NF} \kappa \mathrm{B}$ activation [24]. Therefore, puerarin may also inhibit $\mathrm{NF} \kappa \mathrm{B}$ activation through inhibition of interaction of CD36 with TLR4.

Macrophage apoptosis is a prominent feature of atherosclerotic plaque development. Macrophage apoptosis occurs throughout atherogenesis [26]. Increasing evidence suggests that advanced lesional macrophage apoptosis is associated with the development of vulnerable plaque $[26,34]$. Vulnerable plaque increases risk to precipitate acute coronary syndromes like unstable angina and acute myocardial infarction. The present study showed that puerarin inhibited oxLDL-induced early apoptotic cells of macrophages, and the results may imply a potential therapy of puerarin for patients with unstable angina.

In summary, puerarin has a long history for the treatment of cardiovascular and vascular atherosclerotic diseases [10, 28]. The present study demonstrated that, in human THP1 macrophage, puerarin inhibited atherogenic oxLDL-induced macrophage activation and foam cell formation associated with downregulation of scavenger receptor CD36 expression and TLR4/NF $\kappa$ B pathway, suggesting that puerarin has important anti-inflammatory and antiatherogenic properties. The cellular and molecular mechanisms of puerarin's antiatherosclerotic effects need to be further investigated. 
Our studies provide scientific evidence and may expand our armamentarium to use puerarin for prevention and treatment of cardiovascular and atherosclerotic diseases.

\section{Conflict of Interests}

All authors declare no conflict of interests.

\section{Acknowledgment}

This work was supported by the grant from the National Natural Science Foundation of China (no. 81470532) to MingSheng Zhou.

\section{References}

[1] C. Weber and H. Noels, "Atherosclerosis: current pathogenesis and therapeutic options," Nature Medicine, vol. 17, no. 11, pp. 1410-1422, 2011.

[2] S. Bangalore, Y. Guo, Z. Samadashvili, S. Blecker, J. Xu, and E. L. Hannan, "Everolimus-eluting stents or bypass surgery for multivessel coronary disease," The New England Journal of Medicine, vol. 372, no. 13, pp. 1213-1222, 2015.

[3] I.-C. Hwang, J.-Y. Jeon, Y. Kim et al., "Statin therapy is associated with lower all-cause mortality in patients with nonobstructive coronary artery disease," Atherosclerosis, vol. 239, no. 2, pp. 335-342, 2015.

[4] F. Linden, G. Domschke, C. Erbel, M. Akhavanpoor, H. A. Katus, and C. A. Gleissner, "Inflammatory therapeutic targets in coronary atherosclerosis-from molecular biology to clinical application," Frontiers in Physiology, vol. 5, article 455, 2014.

[5] Q. Liu, J. Li, A. Hartstone-Rose et al., "Chinese herbal compounds for the prevention and treatment of atherosclerosis: experimental evidence and mechanisms," Evidence-Based Complementary and Alternative Medicine, vol. 2015, Article ID 752610, 15 pages, 2015.

[6] S. Colin, G. Chinetti-Gbaguidi, and B. Staels, "Macrophage phenotypes in atherosclerosis," Immunological Reviews, vol. 262, no. 1, pp. 153-166, 2014.

[7] K. Ley, Y. I. Miller, and C. C. Hedrick, "Monocyte and macrophage dynamics during atherogenesis," Arteriosclerosis, Thrombosis, and Vascular Biology, vol. 31, no. 7, pp. 1506-1516, 2011.

[8] D. A. Chistiakov, Y. V. Bobryshev, N. G. Nikiforov, N. V. Elizova, I. A. Sobenin, and A. N. Orekhov, "Macrophage phenotypic plasticity in atherosclerosis: the associated features and the peculiarities of the expression of inflammatory genes," International Journal of Cardiology, vol. 184, pp. 436-445, 2015.

[9] Y. M. Park, M. Febbraio, and R. L. Silverstein, "CD36 modulates migration of mouse and human macrophages in response to oxidized LDL and may contribute to macrophage trapping in the arterial intima," The Journal of Clinical Investigation, vol. 119, no. 1, pp. 136-145, 2009.

[10] K. H. Wong, G. Q. Li, K. M. Li, V. Razmovski-Naumovski, and K. Chan, "Kudzu root: traditional uses and potential medicinal benefits in diabetes and cardiovascular diseases," Journal of Ethnopharmacology, vol. 134, no. 3, pp. 584-607, 2011.

[11] Y. Yuan, J. Zong, H. Zhou et al., "Puerarin attenuates pressure overload-induced cardiac hypertrophy," Journal of Cardiology, vol. 63, no. 1, pp. 73-81, 2014.
[12] Y. P. Hwang, H. G. Kim, T. T. Hien, M. H. Jeong, T. C. Jeong, and H. G. Jeong, "Puerarin activates endothelial nitric oxide synthase through estrogen receptor-dependent PI3-kinase and calcium-dependent AMP-activated protein kinase," Toxicology and Applied Pharmacology, vol. 257, no. 1, pp. 48-58, 2011.

[13] D. K. Y. Yeung, S. W. S. Leung, Y. C. Xu, P. M. Vanhoutte, and R. Y. K. Man, "Puerarin, an isoflavonoid derived from Radix puerariae, potentiates endothelium-independent relaxation via the cyclic AMP pathway in porcine coronary artery," European Journal of Pharmacology, vol. 552, no. 1-3, pp. 105-111, 2006.

[14] J. Kim, K. M. Kim, C.-S. Kim et al., "Puerarin inhibits the retinal pericyte apoptosis induced by advanced glycation end products in vitro and in vivo by inhibiting NADPH oxidaserelated oxidative stress," Free Radical Biology \& Medicine, vol. 53, no. 2, pp. 357-365, 2012.

[15] L. Bao, Y. Zhang, G. Wei et al., "The anti-atherosclerotic effects of puerarin on induced-atherosclerosis in rabbits," Biomedical Papers of the Medical Faculty of the University Palacky, Olomouc, Czechoslovakia, vol. 159, no. 1, pp. 53-59, 2015.

[16] R. Fu, Y. Zhang, Y. Guo, Y. Zhang, Y. Xu, and F. Chen, "Digital gene expression analysis of the pathogenesis and therapeutic mechanisms of ligustrazine and puerarin in rat atherosclerosis," Gene, vol. 552, no. 1, pp. 75-80, 2014.

[17] S. Bekkering, J. Quintin, L. A. B. Joosten, J. W. M. van der Meer, M. G. Netea, and N. P. Riksen, "Oxidized low-density lipoprotein induces long-term proinflammatory cytokine production and foam cell formation via epigenetic reprogramming of monocytes," Arteriosclerosis, Thrombosis, and Vascular Biology, vol. 34, no. 8, pp. 1731-1738, 2014.

[18] L. Chávez-Sánchez, M. G. Garza-Reyes, J. E. Espinosa-Luna, K. Chávez-Rueda, M. V. Legorreta-Haquet, and F. Blanco-Favela, "The role of TLR2, TLR4 and CD36 in macrophage activation and foam cell formation in response to oxLDL in humans," Human Immunology, vol. 75, no. 4, pp. 322-329, 2014.

[19] M.-S. Zhou, K. Chadipiralla, A. J. Mendez et al., "Nicotine potentiates proatherogenic effects of oxLDL by stimulating and upregulating macrophage CD36 signaling," American Journal of Physiology-Heart and Circulatory Physiology, vol. 305, no. 4, pp. H563-H574, 2013.

[20] J. Han, D. P. Hajjar, M. Febbraio, and A. C. Nicholson, "Native and modified low density lipoproteins increase the functional expression of the macrophage class B scavenger receptor, CD36," The Journal of Biological Chemistry, vol. 272, no. 34, pp. 21654-21659, 1997.

[21] S. Gordon and P. R. Taylor, "Monocyte and macrophage heterogeneity," Nature Reviews Immunology, vol. 5, no. 12, pp. 953-964, 2005.

[22] N. Leitinger and I. G. Schulman, "Phenotypic polarization of macrophages in atherosclerosis," Arteriosclerosis, Thrombosis, and Vascular Biology, vol. 33, no. 6, pp. 1120-1126, 2013.

[23] D. M. Mosser and J. P. Edwards, "Exploring the full spectrum of macrophage activation," Nature Reviews Immunology, vol. 8, no. 12, pp. 958-969, 2008.

[24] C. R. Stewart, L. M. Stuart, K. Wilkinson et al., "CD36 ligands promote sterile inflammation through assembly of a Toll-like receptor 4 and 6 heterodimer," Nature Immunology, vol. 11, no. 2, pp. 155-161, 2010.

[25] S. Ghosh and M. S. Hayden, "New regulators of NF- $\kappa$ B in inflammation," Nature Reviews Immunology, vol. 8, no. 11, pp. 837-848, 2008. 
[26] T. Seimon and I. Tabas, "Mechanisms and consequences of macrophage apoptosis in atherosclerosis," Journal of Lipid Research, vol. 50, supplement, pp. S382-S387, 2009.

[27] L. Tang, D. Liu, X. Yi et al., "The protective effects of puerarin in cardiomyocytes from anoxia/reoxygenation injury are mediated by PKCE," Cell Biochemistry and Function, vol. 32, no. 4, pp. 378-386, 2014.

[28] A. K. Maji, S. Pandit, P. Banerji, and D. Banerjee, "Pueraria tuberosa: a review on its phytochemical and therapeutic potential," Natural Product Research, vol. 28, no. 23, pp. 2111-2127, 2014.

[29] M.-H. Bao, Y.-W. Zhang, X.-Y. Lou, Y. Xiao, Y. Cheng, and H.-H. Zhou, "Puerarin protects endothelial cells from oxidized low density lipoprotein induced injuries via the suppression of LOX-1 and induction of eNOS," Canadian Journal of Physiology and Pharmacology, vol. 92, no. 4, pp. 299-306, 2014.

[30] I. Tabas, K. J. Williams, and J. Borén, "Subendothelial lipoprotein retention as the initiating process in atherosclerosis: update and therapeutic implications," Circulation, vol. 116, no. 16, pp. 1832-1844, 2007.

[31] S. O. Rahaman, D. J. Lennon, M. Febbraio, E. A. Podrez, S. L. Hazen, and R. Silverstein, "A CD36-dependent signaling cascade is necessary for macrophage foam cell formation," Cell Metabolism, vol. 4, no. 3, pp. 211-221, 2006.

[32] F. Zhou, L. Wang, P. P. Liu et al., "Puerarin protects brain tissue against cerebral ischemia/reperfusion injury by inhibiting the inflammatory response," Neural Regeneration Research, vol. 9, no. 23, pp. 2074-2080, 2014.

[33] A. R. Brasier, "The nuclear factor-B-interleukin-6 signalling pathway mediating vascular inflammation," Cardiovascular Research, vol. 86, no. 2, pp. 211-218, 2010.

[34] I. Tabas, "Macrophage apoptosis in atherosclerosis: consequences on plaque progression and the role of endoplasmic reticulum stress," Antioxidants \& Redox Signaling, vol. 11, no. 9, pp. 2333-2339, 2009. 


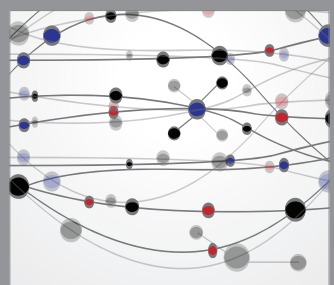

The Scientific World Journal
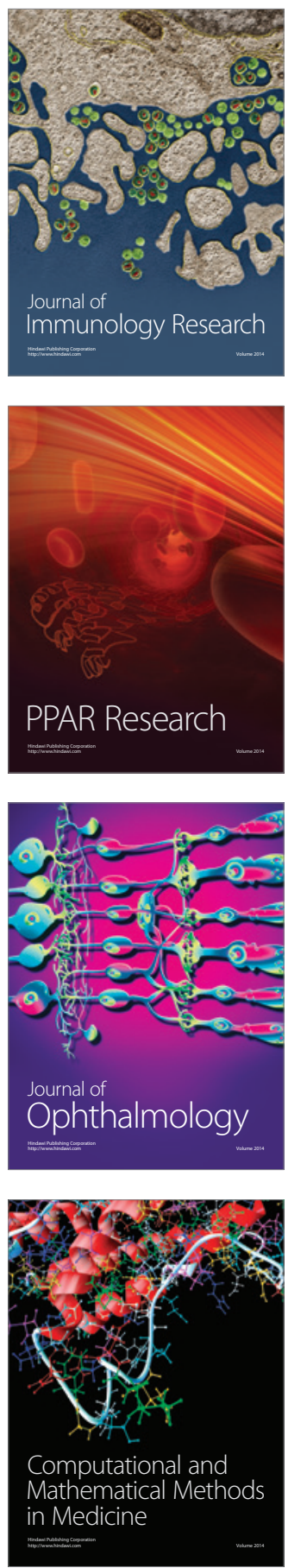

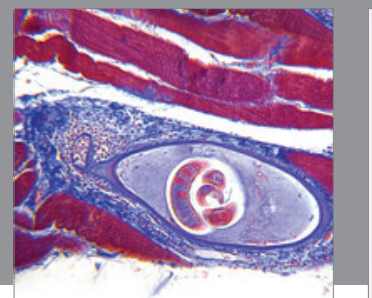

Gastroenterology

Research and Practice
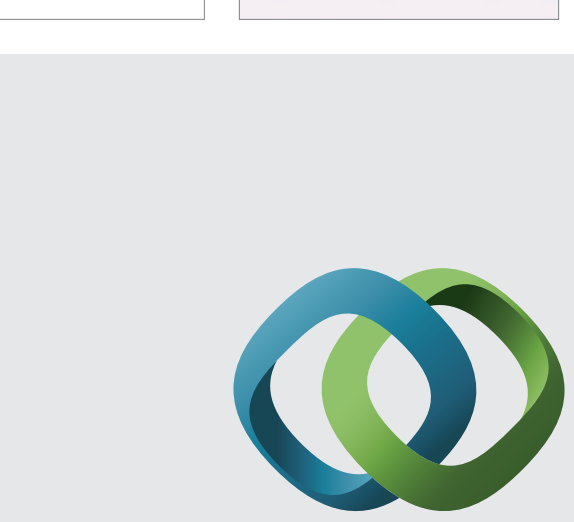

\section{Hindawi}

Submit your manuscripts at

http://www.hindawi.com
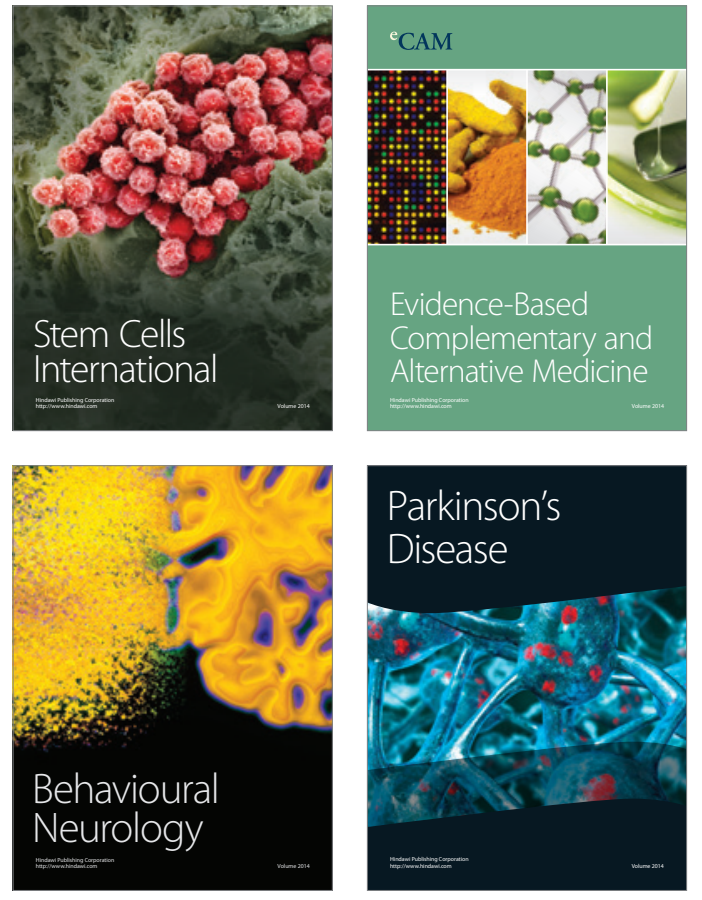
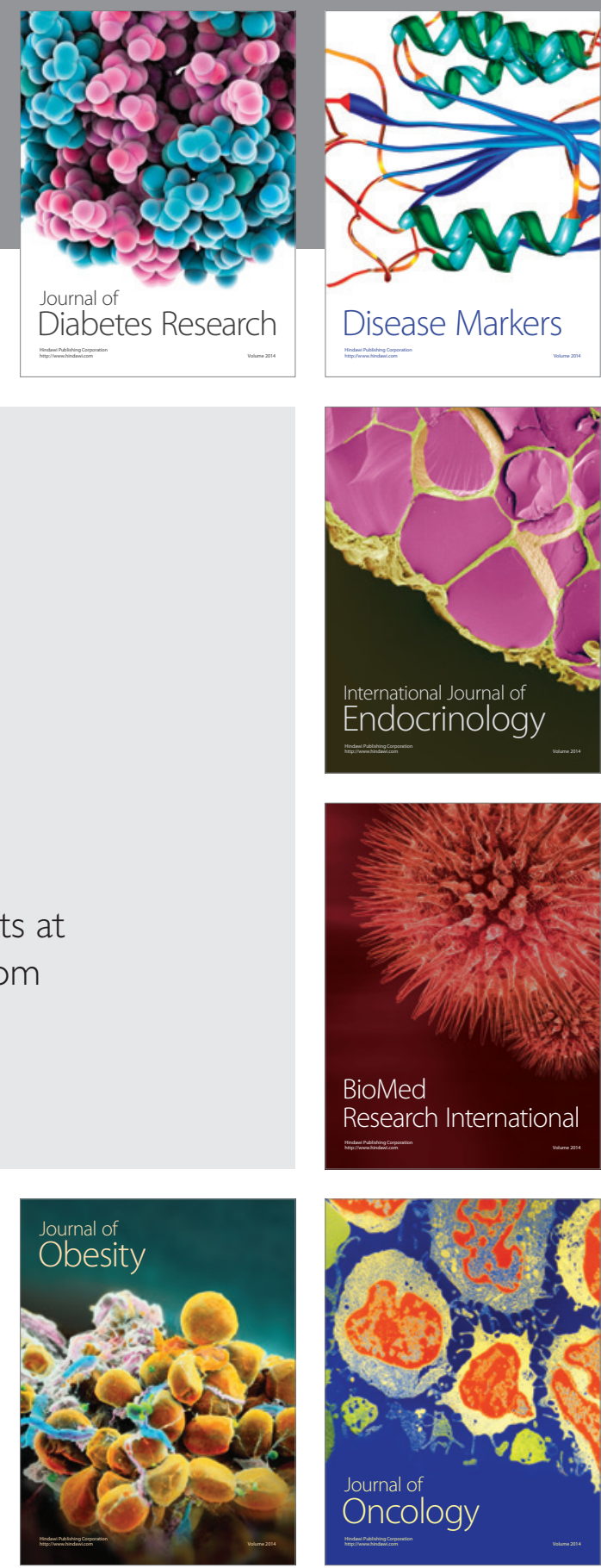

Disease Markers
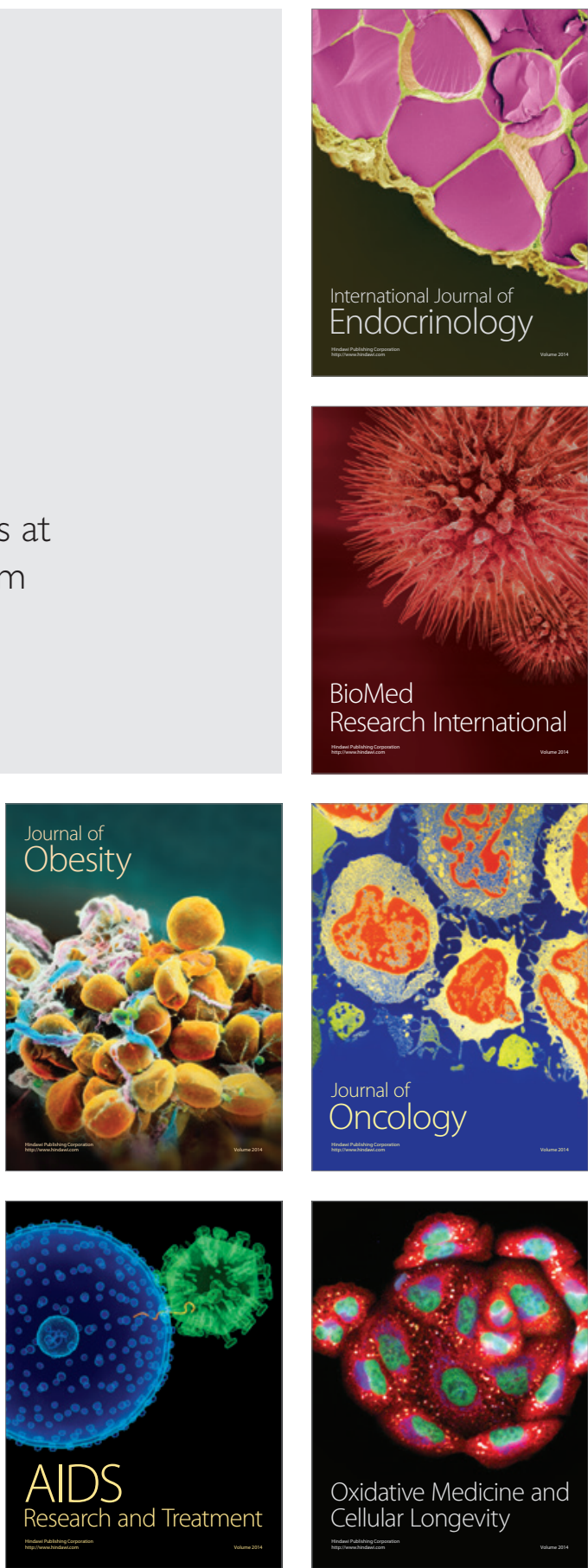